\title{
Synthesis and Bioactivity of Natural Occurring Petasin-Like Derivatives as Antitumor Agents
}

\author{
Xiaolong Shi', , Saiyang Zhang1,2, Jianghao Wang1,2, Tingyu Li, ${ }^{1,2}$, Junju Liư ${ }^{1,2}$, Yi Hou, \\ Lei Liu ${ }^{1,2}$, Dongjun Fu ${ }^{1,2}$, Haiwei Xu1,2, Hongmin Liu ${ }^{1,2}$, Yanbing Zhang1,2* \\ ${ }^{1}$ School of Pharmaceutical Science, Zhengzhou University, Zhengzhou, China \\ ${ }^{2}$ Collaborative Innovation Center of New Drug Research and Safety Evaluation, Henan Province, Zhengzhou, \\ China \\ Email: ${ }^{*}$ zhangyb@zzu.edu.cn
}

Received 6 May 2015; accepted 5 June 2015; published 8 June 2015

Copyright (C) 2015 by authors and Scientific Research Publishing Inc.

This work is licensed under the Creative Commons Attribution International License (CC BY). http://creativecommons.org/licenses/by/4.0/

c) (i) Open Access

\begin{abstract}
Petasin is a potential antitumor against human neuroblastoma cell SK-N-SH by inhibiting the ERK1/2 phosphorylation. In view of its great activity and new antiproliferative mechanisms, a series of petasin derivatives were designed and synthesized, which showed great antiproliferative activity. Among them compounds $1 \mathrm{~h}$ and $1 \mathrm{f}$ were more effective against SK-N-SH cells than petasin with the $\mathrm{IC}_{50}$ values of 0.87 and $2.63 \mu \mathrm{M}$, respectively.
\end{abstract}

Keywords

Petasin, SK-N-SH, Mechanism, Derivative, Antiproliferative Activity

\section{Introduction}

Neuroblastoma (neuroblastoma, NB) [1] is the most common extracranial tumor in infants and young children. Recent clinical statistics show that the neuroblastoma has become the third most common cause of death after renal tumor and leukemia during the childhood [2] [3]. Due to the diversity of clinical manifestations, its treatment varies accordingly. In general, neuroblastoma needs surgery and radioactive therapy, chemotherapy and other treatments are also used for the residual tumors.

Petasin (Figure 1) is the active component of Petasites hybridus L. (Petasites Tricholobus, Compositae), which has been reported to possess many bioactivities including analgesic, anti-inflammation, and inhibition of ileum

"Corresponding author.

How to cite this paper: Shi, X.L., Zhang, S.Y., Wang, J.H., Li, T.Y., Liu, J.J., Hou, Y., Liu, L., Fu, D.J., Xu, H.W., Liu, H.M. and Zhang, Y.B. (2015) Synthesis and Bioactivity of Natural Occurring Petasin-Like Derivatives as Antitumor Agents. Open Journal of Medicinal Chemistry, 5, 23-31. http://dx.doi.org/10.4236/ojmc.2015.52003 
<smiles>C=C(C)[C@H]1C[C@]2(C)C(=CC1=O)CC[C@H](OC(=O)/C(C)=C\C)C2C</smiles><smiles>[R]C(=O)O[C@H]1CCC2=CC(=O)C(=C(C)C)C[C@@]2(C)[C@H]1[Y16]</smiles>

1a: $\mathrm{R}_{1}=4-\mathrm{CH}_{3}-\mathrm{C}_{6} \mathrm{H}_{4}$

1b: $\mathrm{R}_{1}=4-\mathrm{OCH}_{3}-\mathrm{C}_{6} \mathrm{H}_{4}$

1c: $\mathrm{R}_{1}=3-\mathrm{NO}_{2}-\mathrm{C}_{6} \mathrm{H}_{4}$

1d: $\mathrm{R}_{1}=4-\mathrm{NO}_{2}-\mathrm{C}_{6} \mathrm{H}_{4}$

1e: $\mathrm{R}_{1}=3-\mathrm{F}-\mathrm{C}_{6} \mathrm{H}_{4}$

1f: $\mathrm{R}_{1}=2-\mathrm{OH}-\mathrm{C}_{6} \mathrm{H}_{4}$

1g: $\mathrm{R}_{1}=4-\mathrm{Cl}-\mathrm{C}_{6} \mathrm{H}_{4}$

1h: $\mathrm{R}_{1}=2-\mathrm{OCH}_{3}-\mathrm{C}_{6} \mathrm{H}_{4}$<smiles>[R2]OC(=O)/C=C/C(=O)O[C@H]1CCC2=CC(=O)C(=C(C)C)C[C@@]2(C)[C@H]1[Z6]</smiles>

4a: $\mathrm{R}_{2}=\mathrm{CH}_{2}=\mathrm{CHCH}_{2}$

4b: $\mathrm{R}_{2}=\mathrm{CH}_{3} \mathrm{CH}_{2} \mathrm{CH}_{2}$<smiles>[R]C(=O)O[C@H]1CCC2=CC(=O)[C@]3(C[C@]21C)OC3(C)C</smiles>

2a: $\mathrm{R}_{1}=-\mathrm{C}_{6} \mathrm{H}_{4}$

2b: $\mathrm{R}_{1}=4-\mathrm{OCH}_{3}-\mathrm{C}_{6} \mathrm{H}_{4}$

2c: $\mathrm{R}_{1}=3-\mathrm{NO}_{2}-\mathrm{C}_{6} \mathrm{H}_{4}$

2d: $\mathrm{R}_{1}=2-\mathrm{F}-\mathrm{C}_{6} \mathrm{H}_{4}$

2e: $\mathrm{R}_{1}=3-\mathrm{OCH}_{3}-\mathrm{C}_{6} \mathrm{H}_{4} \mathrm{CH}_{2}$<smiles>[R3]C(=O)N[C@H]1CCC2=CC(=O)C(=C(C)C)C[C@@]2(C)[C@@H]1[18OH]</smiles>

8a: $\mathrm{R}_{3}=\mathrm{CH}_{3}$

8b: $\mathrm{R}_{3}=\mathrm{CH}_{2} \mathrm{Cl}$

8c: $\mathrm{R}_{3}=4-\mathrm{Cl}-\mathrm{C}_{6} \mathrm{H}_{4}$

Figure 1 . The structures of petasin and petasin derivatives $\left(R_{1}, R_{2}, R_{3}=\right.$ alkyl group).

contraction. In our group, the great antitumor activity against Sk-N-SH was reported for the first time [4]. Petasin successfully inhibited the proliferation but not induced the apoptosis. The inhibition of ERK1/2 phosphorylation might be involved in the antiproliferation.

Based on the above results, a series of petasin derivatives were designed and synthesized (Figure 1) for investigating the structure-activity relationship and discovering new antiproliferative agents.

\section{Chemistry}

Petasin derivatives were efficiently synthesized as described in Scheme 1. Compounds 1a-h and $\mathbf{3}$ were obtained from the isopetasinol by acylation reactions with carboxylic acids or maleic anhydride [5] [6]. Isopetasinol was prepared by the hydrolysis [7] of petasin in the presence of $\mathrm{NaOH}$. Compounds 2 were prepared by an epoxidation [8]-[10] of compounds $\mathbf{1}$. Compounds $\mathbf{4 a - b}$ with an unsaturated side chain were synthesized by the reaction of compound $\mathbf{3}$ and different alkyl halides.

To further investigate SARs, compounds 8a-c with an amide functional group were designed and synthesized [11]-[17]. Tosylation of isopetasinol resulted in compound $\mathbf{5}$, followed by nucleophilic substitution with $\mathrm{NaN}_{3}$ giving the corresponding azide $\mathbf{6}$. Zn-mediated reduction of compound $\mathbf{6}$ produced compound $\mathbf{7}$, which was then subjected to prepare compounds $\mathbf{8 a - c}$ by acylation reactions.

\section{Results and Discussion}

The $\mathrm{IC}_{50}$ values for all obtained petasin analogues against three human cancer cell lines including SK-N-SH, MGC-803 and HepG-2 were determined using MTT assay [18]-[22]. Natural occurring petasin, isopetasinol and cisplatin were used as positive controls. The results were demonstrated in Table 1. The cell-based investigation demonstrated that some synthetic compounds showed much more potent or comparable antiproliferative activity compared to the petasin. Especially they showed selective antiproliferative activities toward SK-N-SH cells with weak inhibition against other tumor cells.

Compared to petasin, the antiproliferative activities of isopetasinol against the tested cancer cell lines was decreased significantly ( $\mathrm{IC}_{50}>100 \mu \mathrm{M}$ ), which indicated that an additional unsaturated ester group is beneficial for the activity. Therefore, substituted benzoic ester group was introduced as represented in compounds 1a-h. Interestingly, some of these compounds showed great cytotoxicity against SK-N-SH and were much more potent than petasin and cisplatin. Compounds with electron-donating groups attached to the phenyl ring were much 


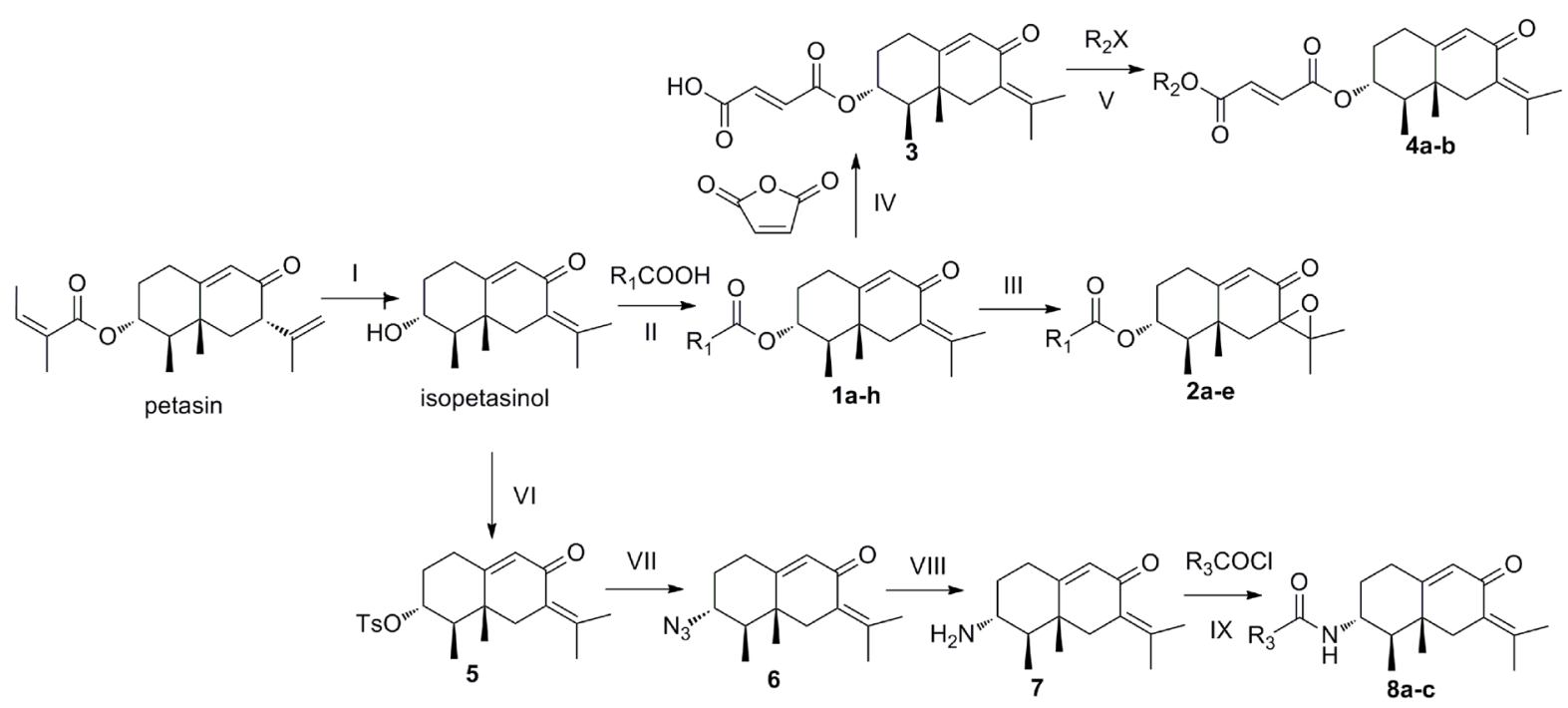

Scheme 1. Reagents and conditions: 1) $\left.\left.\mathrm{NaOH}, \mathrm{CH}_{3} \mathrm{OH}, 62^{\circ} \mathrm{C}, 4-6 \mathrm{~h} ; 2\right) \mathrm{DMAP}, \mathrm{DCC}, \mathrm{CH}_{2} \mathrm{Cl}_{2}, 0^{\circ} \mathrm{C}, 6 \mathrm{~h} ; 3\right) \mathrm{m}-$ $\mathrm{ClC}_{6} \mathrm{H}_{5} \mathrm{COOOH}, \mathrm{CH}_{2} \mathrm{Cl}_{2}$, rt, $3 \mathrm{~h}$; 4) $\left(\mathrm{C}_{2} \mathrm{H}_{5}\right)_{3} \mathrm{~N}, \mathrm{CH}_{2} \mathrm{Cl}_{2}, 0^{\circ} \mathrm{C}, 2 \mathrm{~h}$; 5) $\left.\mathrm{Na}_{2} \mathrm{CO}_{3}, \mathrm{DMF}, 65^{\circ} \mathrm{C}, 2 \mathrm{~h} ; 6\right) \mathrm{m}_{-} \mathrm{CH}_{3} \mathrm{C}_{6} \mathrm{H}_{5} \mathrm{SO}_{2} \mathrm{Cl}_{\text {, }}$ $\left(\mathrm{C}_{2} \mathrm{H}_{5}\right)_{3} \mathrm{~N}, \mathrm{CH}_{2} \mathrm{Cl}_{2}, 0^{\circ} \mathrm{C}, 6 \mathrm{~h}$; 7) $\left.\mathrm{NaN}_{3}, \mathrm{DMF}, 95^{\circ} \mathrm{C}, 8 \mathrm{~h} ; 8\right) \mathrm{Zn}, \mathrm{NH}_{4} \mathrm{Cl}, \mathrm{THF} / \mathrm{H}_{2} \mathrm{O}, 2 \mathrm{~h}, \mathrm{rt}$; 9) $\left(\mathrm{C}_{2} \mathrm{H}_{5}\right)_{3} \mathrm{~N} \mathrm{CH}_{2} \mathrm{Cl}_{2}, 0^{\circ} \mathrm{C}, 2 \mathrm{~h}$.

Table 1. In vitro inhibitory activity of petasin derivatives against SK-N-SH, MGC-803 and HepG-2 cell lines.

\begin{tabular}{|c|c|c|c|}
\hline \multirow{2}{*}{ Compd } & \multicolumn{3}{|c|}{$\mathrm{IC}_{50}(\mu \mathrm{mol} / \mathrm{L})$} \\
\hline & SK-N-SH & MGC-803 & HepG-2 \\
\hline 1a & $12.01 \pm 1.27$ & $>100$ & $38.02 \pm 1.87$ \\
\hline $1 b$ & $5.98 \pm 1.06$ & $12.99 \pm 2.62$ & $64.17 \pm 2.06$ \\
\hline 1c & $14.91 \pm 1.85$ & $12.84 \pm 1.87$ & $34.55 \pm 3.51$ \\
\hline 1d & $>100$ & $13.73 \pm 2.46$ & $30.30 \pm 2.43$ \\
\hline $\begin{array}{l}\text { 1e } \\
1 \text { f } \\
1 \mathrm{~g} \\
1 \mathrm{~h}\end{array}$ & $\begin{array}{c}31.36 \pm 2.01 \\
2.63 \pm 0.76 \\
61.25 \pm 3.21 \\
0.87 \pm 0.28\end{array}$ & $\begin{array}{c}33.84 \pm 2.85 \\
24.83 \pm 1.97 \\
40.40 \pm 3.41 \\
>100\end{array}$ & $\begin{array}{l}33.82 \pm 2.01 \\
57.33 \pm 2.53 \\
32.02 \pm 1.98 \\
27.46 \pm 1.63\end{array}$ \\
\hline $2 a$ & $34.78 \pm 3.56$ & $43.75 \pm 3.78$ & $>100$ \\
\hline $\begin{array}{l}2 b \\
2 c\end{array}$ & $\begin{array}{l}76.23 \pm 2.18 \\
26.87 \pm 1.87\end{array}$ & $\begin{array}{l}>100 \\
>100\end{array}$ & $\begin{array}{c}>100 \\
49.34 \pm 1.76\end{array}$ \\
\hline 2d & $48.95 \pm 2.14$ & $>100$ & $>100$ \\
\hline $2 e$ & $>100$ & $>100$ & $>100$ \\
\hline $4 a$ & $14.54 \pm 1.43$ & $43.19 \pm 2.86$ & $58.17 \pm 3.01$ \\
\hline $4 b$ & $12.76 \pm 1.52$ & $>100$ & $>100$ \\
\hline $\mathbf{8 a}$ & $>100$ & $>100$ & $>100$ \\
\hline $8 \mathbf{b}$ & $31.80 \pm 2.07$ & $36.86 \pm 3.41$ & $27.63 \pm 2.43$ \\
\hline $8 c$ & $64.31 \pm 2.65$ & $78.37 \pm 4.01$ & $43.98 \pm 3.01$ \\
\hline Isopetasinol & $>100$ & $>100$ & $>100$ \\
\hline $\begin{array}{c}\text { Petasin } \\
\text { Cisplatin }\end{array}$ & $\begin{array}{c}5.76 \pm 0.98 \\
12.32 \pm 1.06\end{array}$ & $\begin{array}{l}34.41 \pm 2.75 \\
10.88 \pm 1.86\end{array}$ & $\begin{array}{c}26.84 \pm 1.65 \\
8.14 \pm 0.89\end{array}$ \\
\hline
\end{tabular}

more potent than those with electron-withdrawing groups. Among them, compound $\mathbf{1 h}$ and $\mathbf{1 f}$ showed the best cytotoxicity with the $\mathrm{IC}_{50}$ values of 0.87 and $2.63 \mu \mathrm{M}$, respectively, which were about 14 or 15 fold more potent than cisplatin $\left(\mathrm{IC}_{50}=12.32 \mu \mathrm{M}\right)$. 
Compounds 2a-e with epoxy ring showed bad antiproliferative activity against SK-N-SH with the IC I0 $_{50}$ values more than $26 \mu \mathrm{M}$ and were less potent than cisplatin and petasin $\left(\mathrm{IC}_{50}=5.76 \mu \mathrm{M}\right)$, which indicated that exodouble bond were important for the antiproliferative activity. Besides, the antiproliferative activity of compounds $\mathbf{4 a - b}$ and compounds $\mathbf{8 a - c}$ also become to be worse than that of compounds 1a-1h and the positive control.

In conclusion, SAR of new petasin derivatives against SK-N-SH showed that an additional ester group and the exo-double bond were important for the antiproliferative activity. Furthermore the isomerization of the double bond and unsaturated ester unaffected the antiproliferative activity. But if the ester group was substituted with amide group, the antiproliferative activity against Sk-N-SH showed a certain extent effects. And compounds $\mathbf{1 h}$ and $\mathbf{1 f}$ were discovered, which showed selective and great antiproliferative activity with $\mathrm{IC}_{50}$ values of 0.87 and $2.63 \mu \mathrm{M}$ against SK-N-SH cells. The result in this study is valuable for the design and optimization of petasin derivative and the discovery of new antiproliferative agent.

\section{Experimental Section}

\subsection{Chemistry}

Petasin, extracted from Ligularia fischeri with traditional ethanol reflux. Reagents and solvents were purchased from commercial sources and were used without further purification. Thin-layer chromatography (TLC) was performed on silica gel $\mathrm{GF}_{254}$ plates. Silicagel $\mathrm{GF}_{254}$ and H (200 - 300 mesh) from Qingdao Haiyang Chemical Company was used for TLC, preparative TLC, and column chromatography respectively. Melting points were determined on an X-5 micromelting apparatus and are uncorrected. ${ }^{1} \mathrm{H}$ NMR and ${ }^{13} \mathrm{C}$ NMR spectra were recorded on a Bruker $400 \mathrm{MHz}$ and $100 \mathrm{MHz}$ spectrometer respectively. High resolution mass spectra (HRMS) were recorded on a Waters Micromass Q-T of Micromass spectrometer by electrospray ionization (ESI).

\subsubsection{General Procedure for the Synthesis of Compounds 1a-h}

A mixture of isopetasinol (1 equiv) and Carboxylic acid (1.5 equiv) in $\mathrm{CH}_{2} \mathrm{Cl}_{2}$ in the presence of DMAP (1 equiv) and DCC (1 equiv) was stirred at $0^{\circ} \mathrm{C}$ for $6 \mathrm{~h}$. The disappearance of compound isopetasinol was monitored by TLC. Upon completion, the solvent was removed and water was added, the reaction mixture was extracted with $\mathrm{CH}_{2} \mathrm{Cl}_{2}$. The combined organic layer was washed with brine, dried over anhydrous $\mathrm{MgSO}_{4}$ and concentrated under vacuum to afford the crude product. The crude product was purified by a flash column to yield the pure product 1a-h.

1,8a-dimethyl-6-oxo-7-(propan-2-ylidene)-1,2,3,4,6,7,8,8a-octahydronaphthalen-2-yl-4-methylbenzoate (1a). Yellow solid, yield $75 \%$, mp: $71.2^{\circ} \mathrm{C}-72.1^{\circ} \mathrm{C},{ }^{1} \mathrm{H}$ NMR $\left(400 \mathrm{MHz}, \mathrm{CDCl}_{3}\right) \delta 7.94-7.25$ (m, $\left.4 \mathrm{H}, \mathrm{ArH}\right)$, $5.81(\mathrm{~s}, 1 \mathrm{H}, \mathrm{HC}=\mathrm{C}), 5.14-5.01(\mathrm{~m}, 1 \mathrm{H}, \mathrm{CH}), 2.96$ (d, $\left.J=13.7 \mathrm{~Hz}, 1 \mathrm{H}, \mathrm{CH}_{2}\right), 2.52\left(\mathrm{~d}, J=13.6,1 \mathrm{H}, \mathrm{CH}_{2}\right), 2.42$ (s, 3H, $\mathrm{CH}_{3}$ ), $2.39-2.26\left(\mathrm{~m}, 2 \mathrm{H}, \mathrm{CH}_{2}\right), 2.23$ (d, J = $\left.11.0 \mathrm{~Hz}, 1 \mathrm{H}, \mathrm{CH}_{2}\right), 2.11\left(\mathrm{~s}, 3 \mathrm{H}, \mathrm{CH}_{3}\right), 1.87\left(\mathrm{~s}, 3 \mathrm{H}, \mathrm{CH}_{3}\right)$, $1.81\left(\mathrm{~d}, J=11.0,1 \mathrm{H}, \mathrm{CH}_{2}\right), 1.63-1.54(\mathrm{~m}, 1 \mathrm{H}, \mathrm{CH}), 1.08\left(\mathrm{~s}, 3 \mathrm{H}, \mathrm{CH}_{3}\right), 1.04\left(\mathrm{~d}, J=6.7 \mathrm{~Hz}, 3 \mathrm{H}, \mathrm{CH}_{3}\right) .{ }^{13} \mathrm{C} \mathrm{NMR}^{\mathrm{N}}$ $\left(100 \mathrm{MHz}, \mathrm{CDCl}_{3}\right) \delta$ 206.94, 191.66, 166.23, 165.15, 143.73, 143.43, 129.60, 129.11, 127.59, 127.14, 126.76, 74.01, 46.34, 42.27, 41.17, 31.65, 30.91, 30.13, 22.61, 22.13, 21.65, 17.19, 10.83. HR-MS (ESI): Calcd. $\mathrm{C}_{23} \mathrm{H}_{28} \mathrm{O}_{3},[\mathrm{M}+\mathrm{H}]^{+} \mathrm{m} / \mathrm{z}$ : 352.2126, found: 352.2128.

1,8a-dimethyl-6-oxo-7-(propan-2-ylidene)-1,2,3,4,6,7,8,8a-octahydronaphthalen-2-yl-4-methoxybenzoate (1b). Yellow solid, yield 70\%, mp: $107.7^{\circ} \mathrm{C}-110.4^{\circ} \mathrm{C},{ }^{1} \mathrm{H}$ NMR $\left(400 \mathrm{MHz}, \mathrm{CDCl}_{3}\right) \delta 8.03-6.95$ (m, $\left.4 \mathrm{H}, \mathrm{ArH}\right)$, $5.83(\mathrm{~s}, 1 \mathrm{H}, \mathrm{HC}=\mathrm{C}), 5.13-5.04(\mathrm{~m}, 1 \mathrm{H}, \mathrm{CH}), 3.89\left(\mathrm{~s}, 3 \mathrm{H}, \mathrm{CH}_{3}\right), 2.98\left(\mathrm{~d}, J=13.7 \mathrm{~Hz}, 1 \mathrm{H}, \mathrm{CH}_{2}\right), 2.56(\mathrm{~d}, J=$ 13.7, 1H, $\mathrm{CH}_{2}$ ), $2.46-2.29\left(\mathrm{~m}, 2 \mathrm{H}, \mathrm{CH}_{2}\right), 2.25$ (d, J = $\left.11.0 \mathrm{~Hz}, 1 \mathrm{H}, \mathrm{CH}_{2}\right), 2.13\left(\mathrm{~s}, 3 \mathrm{H}, \mathrm{CH}_{3}\right), 1.89\left(\mathrm{~s}, 3 \mathrm{H}, \mathrm{CH}_{3}\right)$, $1.82\left(\mathrm{~d}, J=11.0,1 \mathrm{H}, \mathrm{CH}_{2}\right), 1.63-1.57(\mathrm{~m}, 1 \mathrm{H}, \mathrm{CH}), 1.10\left(\mathrm{~s}, 3 \mathrm{H}, \mathrm{CH}_{3}\right), 1.06\left(\mathrm{~d}, J=6.7 \mathrm{~Hz}, 3 \mathrm{H}, \mathrm{CH}_{3}\right) .{ }^{13} \mathrm{C} \mathrm{NMR}^{\mathrm{N}}$ $\left(100 \mathrm{MHz}_{\mathrm{CDCl}}\right) \delta$ 206.99, 191.71, 165.93, 165.26, 163.45, 143.46, 131.59, 127.14, 126.72, 122.71, 113.65, 73.85, 55.46, 46.38, 42.28, 41.17, 31.70, 30.91, 30.15, 22.62, 22.14, 17.19, 10.83. HR-MS (ESI): Calcd. $\mathrm{C}_{23} \mathrm{H}_{28} \mathrm{O}_{4},[\mathrm{M}+\mathrm{H}]^{+} \mathrm{m} / \mathrm{z}$ : 368.2047, found: 368.2049.

1,8a-dimethyl-6-oxo-7-(propan-2-ylidene)-1,2,3,4,6,7,8,8a-octahydronaphthalen-2-yl-3-nitrobenzoate (1c). White solid, yield $74 \%$, mp: $128.9^{\circ} \mathrm{C}-132.8^{\circ} \mathrm{C},{ }^{1} \mathrm{H}$ NMR (400 MHz, $\left.\mathrm{CDCl}_{3}\right) \delta 7.95-7.64$ (m, 4H, ArH), $5.82(\mathrm{~s}$, $1 \mathrm{H} \mathrm{HC}=\mathrm{C}), 5.12-5.01(\mathrm{~m}, 1 \mathrm{H}, \mathrm{CH}), 2.95\left(\mathrm{~d}, J=13.7 \mathrm{~Hz}, 1 \mathrm{H}, \mathrm{CH}_{2}\right), 2.56\left(\mathrm{~d}, J=13.7 \mathrm{~Hz}, 1 \mathrm{H}, \mathrm{CH}_{2}\right), 2.47-$ $2.39\left(\mathrm{~m}, 2 \mathrm{H}, \mathrm{CH}_{2}\right), 2.21\left(\mathrm{~d}, J=11.1 \mathrm{~Hz}, 1 \mathrm{H}, \mathrm{CH}_{2}\right), 2.12\left(\mathrm{~s}, 3 \mathrm{H}, \mathrm{CH}_{3}\right), 1.88\left(\mathrm{~s}, 3 \mathrm{H}, \mathrm{CH}_{3}\right), 1.78-1.70(\mathrm{~m}, 1 \mathrm{H}$, $\mathrm{CH}), 1.59$ (d, $\left.J=11.0 \mathrm{~Hz}, 1 \mathrm{H}, \mathrm{CH}_{2}\right), 1.09$ (s, 3H, $\left.\mathrm{CH}_{3}\right), 1.06\left(\mathrm{~d}, J=6.7 \mathrm{~Hz}, 3 \mathrm{H}, \mathrm{CH}_{3}\right) .{ }^{13} \mathrm{C} \mathrm{NMR}(100 \mathrm{MHz}$, $\left.\mathrm{CDCl}_{3}\right) \delta$ 207.02, 191.53, 165.14, 164.52, 147.95, 143.63, 132.98, 131.74, 129.65, 127.88, 126.99, 123.96, 
76.40, 45.85, 42.28, 41.04, 30.95, 29.94, 22.63, 22.16, 17.17, 10.79. HR-MS (ESI): Calcd. $\mathrm{C}_{22} \mathrm{H}_{25} \mathrm{NO}_{5},[\mathrm{M}+\mathrm{H}]^{+}$ $\mathrm{m} / \mathrm{z}$ : 383.1807, found: 383.1809 .

1,8a-dimethyl-6-oxo-7-(propan-2-ylidene)-1,2,3,4,6,7,8,8a-octahydronaphthalen-2-yl-4-nitrobenzoate (1d). White solid yield $75 \%$, mp: $88.1^{\circ} \mathrm{C}-91.4^{\circ} \mathrm{C},{ }^{1} \mathrm{H}$ NMR $\left(400 \mathrm{MHz}, \mathrm{CDCl}_{3}\right) \delta 8.36-8.21(\mathrm{~m}, 4 \mathrm{H}, \mathrm{ArH}), 5.84(\mathrm{~s}$, $1 \mathrm{H}, \mathrm{HC}=\mathrm{C}), 5.17-5.09(\mathrm{~m}, 1 \mathrm{H}, \mathrm{CH}), 2.99\left(\mathrm{~d}, J=13.7 \mathrm{~Hz}, 1 \mathrm{H}, \mathrm{CH}_{2}\right), 2.58\left(\mathrm{~d}, J=13.7 \mathrm{~Hz}, 1 \mathrm{H}, \mathrm{CH}_{2}\right), 2.48-$ $2.31(\mathrm{~m}, 2 \mathrm{H}), 2.26$ (d, $\left.J=11.0 \mathrm{~Hz}, 1 \mathrm{H}, \mathrm{CH}_{2}\right), 2.14\left(\mathrm{~s}, 3 \mathrm{H}, \mathrm{CH}_{3}\right), 1.89$ (s, 3H, $\left.\mathrm{CH}_{3}\right), 1.86\left(\mathrm{~d}, J=11.2,1 \mathrm{H}, \mathrm{CH}_{2}\right)$, $1.72-1.64(\mathrm{~m}, 1 \mathrm{H}, \mathrm{CH}), 1.11\left(\mathrm{~s}, 3 \mathrm{H}, \mathrm{CH}_{3}\right), 1.08\left(\mathrm{~d}, J=6.7 \mathrm{~Hz}, 3 \mathrm{H}, \mathrm{CH}_{3}\right) \cdot{ }^{13} \mathrm{C}$ NMR $\left(100 \mathrm{MHz}, \mathrm{CDCl}_{3}\right) \delta$ 206.86, 191.48, 164.32, 164.29, 150.61, 143.79, 135.67, 130.71, 126.99, 126.92, 123.60, 75.66, 46.16, 42.25, 41.13, 31.52, 30.93, 29.99, 22.65, 22.17, 17.17, 10.93. HR-MS (ESI): Calcd. $\mathrm{C}_{22} \mathrm{H}_{25} \mathrm{NO}_{5}$, $[\mathrm{M}+\mathrm{H}]^{+} \mathrm{m} / \mathrm{z}: 383.1807$, found: 383.1813.

1,8a-dimethyl-6-oxo-7-(propan-2-ylidene)-1,2,3,4,6,7,8,8a-octahydronaphthalen-2-yl-3-fluorobenzoate (1e). White solid, yield $73 \%$, mp: $115.1^{\circ} \mathrm{C}-116.7^{\circ} \mathrm{C},{ }^{1} \mathrm{H}$ NMR $\left(400 \mathrm{MHz}, \mathrm{CDCl}_{3}\right) \delta 7.85-7.27$ (m, 4H, ArH), $5.82(\mathrm{~s}$, $1 \mathrm{H}, \mathrm{HC}=\mathrm{C}), 5.11-5.03(\mathrm{~m}, 1 \mathrm{H}, \mathrm{CH}), 2.98\left(\mathrm{~d}, J=13.7 \mathrm{~Hz}, 1 \mathrm{H}, \mathrm{CH}_{2}\right), 2.57\left(\mathrm{~d}, J=13.7 \mathrm{~Hz}, 1 \mathrm{H}, \mathrm{CH}_{2}\right), 2.46-$ $2.29\left(\mathrm{~m}, 2 \mathrm{H}, \mathrm{CH}_{2}\right), 2.26\left(\mathrm{~d}, J=11.2 \mathrm{~Hz}, 1 \mathrm{H}, \mathrm{CH}_{2}\right), 2.12\left(\mathrm{~s}, 3 \mathrm{H}, \mathrm{CH}_{3}\right), 1.87\left(\mathrm{~s}, 3 \mathrm{H}, \mathrm{CH}_{3}\right), 1.82(\mathrm{~d}, J=11.4,1 \mathrm{H}$, $\left.\mathrm{CH}_{2}\right), 1.61-1.53(\mathrm{~m}, 1 \mathrm{H}, \mathrm{CH}), 1.09$ (s, 3H, $\left.\mathrm{CH}_{3}\right), 1.05$ (d, $\left.J=6.7 \mathrm{~Hz}, 3 \mathrm{H}, \mathrm{CH}_{3}\right) \cdot{ }^{13} \mathrm{C}$ NMR $\left(100 \mathrm{MHz}, \mathrm{CDCl}_{3}\right) \delta$ 206.99, 191.60, 164.90, 143.60, 127.04, 126.87, 125.31, 120.23, 120.01, 116.58, 116.35, 74.83, 46.21, 42.25, 41.13, 31.55, 30.94, 30.05, 22.64, 22.15, 17.17, 10.87. HR-MS (ESI): Calcd. $\mathrm{C}_{22} \mathrm{H}_{25} \mathrm{FO}_{3},[\mathrm{M}+\mathrm{H}]^{+} \mathrm{m} / \mathrm{z}$ : 356.1863, found: 356.1865.

1,8a-dimethyl-6-oxo-7-(propan-2-ylidene)-1,2,3,4,6,7,8,8a-octahydronaphthalen-2-yl-2-hydroxybenzoate (1f). White solid, yield $73 \%$, mp: $128.8^{\circ} \mathrm{C}-134.6^{\circ} \mathrm{C},{ }^{1} \mathrm{H}$ NMR $\left(400 \mathrm{MHz}, \mathrm{CDCl}_{3}\right) \delta 10.81$ (s, $\left.1 \mathrm{H}, \mathrm{OH}\right), 7.84$ $6.89(\mathrm{~m}, 4 \mathrm{H}, \mathrm{ArH}), 5.82(\mathrm{~s}, 1 \mathrm{H}, \mathrm{HC}=\mathrm{C}), 5.14-5.06(\mathrm{~m}, 1 \mathrm{H}, \mathrm{CH}), 2.99\left(\mathrm{~d}, J=13.7 \mathrm{~Hz}, 1 \mathrm{H}, \mathrm{CH}_{2}\right), 2.56(\mathrm{~d}, J=$ $\left.13.7 \mathrm{~Hz}, 1 \mathrm{H}, \mathrm{CH}_{2}\right), 2.48-2.30\left(\mathrm{~m}, 2 \mathrm{H}, \mathrm{CH}_{2}\right), 2.26\left(\mathrm{~d}, J=11.2 \mathrm{~Hz}, 1 \mathrm{H}, \mathrm{CH}_{2}\right), 2.12\left(\mathrm{~s}, 3 \mathrm{H}, \mathrm{CH}_{3}\right), 1.87(\mathrm{~s}, 3 \mathrm{H}$, $\left.\mathrm{CH}_{3}\right), 1.83\left(\mathrm{~d}, J=11.2,5.5 \mathrm{~Hz}, 1 \mathrm{H}, \mathrm{CH}_{2}\right), 1.72-1.62(\mathrm{~m}, 1 \mathrm{H}, \mathrm{CH}), 1.09\left(\mathrm{~s}, 3 \mathrm{H}, \mathrm{CH}_{3}\right), 1.06$ (d, $J=6.7 \mathrm{~Hz}, 3 \mathrm{H}$, $\left.\mathrm{CH}_{3}\right) .{ }^{13} \mathrm{C}$ NMR $\left(100 \mathrm{MHz}, \mathrm{CDCl}_{3}\right) \delta 206.91,191.53,164.42,161.86,143.72,135.89,129.75,126.96,119.23$, 117.68, 112.47, 75.08, 46.18, 42.24, 41.15, 31.58, 30.96, 30.08, 22.66, 22.41, 17.61, 10.97. HR-MS (ESI): Calcd. $\mathrm{C}_{22} \mathrm{H}_{26} \mathrm{O}_{4},[\mathrm{M}+\mathrm{H}]^{+} \mathrm{m} / \mathrm{z}$ : 354.1906, found: 354.1907.

1,8a-dimethyl-6-oxo-7-(propan-2-ylidene)-1,2,3,4,6,7,8,8a-octahydronaphthalen-2-yl-4-chlorobenzoate (1g). Yellow solid, yield 63\%, mp: $69.2^{\circ} \mathrm{C}-72.8^{\circ} \mathrm{C},{ }^{1} \mathrm{H}$ NMR $\left(400 \mathrm{MHz}, \mathrm{CDCl}_{3}\right) \delta 7.98-7.43(\mathrm{~m}, 4 \mathrm{H}, \mathrm{ArH})$, $5.81(\mathrm{~s}, 1 \mathrm{H}, \mathrm{HC}=\mathrm{C}), 5.10-5.02(\mathrm{~m}, 1 \mathrm{H}, \mathrm{CH}), 2.98\left(\mathrm{~d}, J=13.7 \mathrm{~Hz}, 1 \mathrm{H}, \mathrm{CH}_{2}\right), 2.56\left(\mathrm{~d}, J=13.7,1 \mathrm{H}, \mathrm{CH}_{2}\right), 2.44-$ $2.30\left(\mathrm{~m}, 2 \mathrm{H}, \mathrm{CH}_{2}\right), 2.25\left(\mathrm{~d}, J=11.1 \mathrm{~Hz}, 1 \mathrm{H}, \mathrm{CH}_{2}\right), 2.11\left(\mathrm{~s}, 3 \mathrm{H}, \mathrm{CH}_{3}\right), 1.86\left(\mathrm{~s}, 3 \mathrm{H}, \mathrm{CH}_{3}\right), 1.84-1.76(\mathrm{~m}, 1 \mathrm{H}$, $\mathrm{CH}), 1.58\left(\mathrm{~d}, J=11.2 \mathrm{~Hz}, 1 \mathrm{H}, \mathrm{CH}_{2}\right), 1.08\left(\mathrm{~s}, 3 \mathrm{H}, \mathrm{CH}_{3}\right), 1.04\left(\mathrm{~d}, J=6.7 \mathrm{~Hz}, 3 \mathrm{H}, \mathrm{CH}_{3}\right) .{ }^{13} \mathrm{C} \mathrm{NMR}(100 \mathrm{MHz}$, $\left.\mathrm{CDCl}_{3}\right) \delta$ 206.99, 191.61, 165.32, 164.83, 143.62, 139.52, 130.97, 128.77, 128.74, 127.04, 126.84, 74.65, 46.26, 42.26, 41.14, 31.59, 30.92, 30.07, 22.63, 22.15, 17.17, 10.86. HR-MS (ESI): Calcd. $\mathrm{C}_{22} \mathrm{H}_{25} \mathrm{ClO}_{3},[\mathrm{M}+\mathrm{H}]^{+} \mathrm{m} / \mathrm{z}$ : 372.1570, found: 372.1571 .

1,8a-dimethyl-6-oxo-7-(propan-2-ylidene)-1,2,3,4,6,7,8,8a-octahydronaphthalen-2-yl-2-methoxybenzoate (1h). White solid, yield 73\%, mp: $102.1^{\circ} \mathrm{C}-103.7^{\circ} \mathrm{C},{ }^{1} \mathrm{H}$ NMR $\left(400 \mathrm{MHz}, \mathrm{CDCl}_{3}\right) \delta 7.77-6.96$ (m, 4H, ArH), $5.80(\mathrm{~s}, 1 \mathrm{H} \mathrm{HC}=\mathrm{C}), 5.11-5.01(\mathrm{~m}, 1 \mathrm{H}, \mathrm{CH}), 3.91\left(\mathrm{~s}, 3 \mathrm{H}, \mathrm{CH}_{3}\right), 2.97\left(\mathrm{~d}, J=13.7 \mathrm{~Hz}, 1 \mathrm{H}, \mathrm{CH}_{2}\right), 2.56(\mathrm{~d}, J=$ 13.7, $\left.1 \mathrm{H}, \mathrm{CH}_{2}\right), 2.45-2.31\left(\mathrm{~m}, 2 \mathrm{H}, \mathrm{CH}_{2}\right), 2.24\left(\mathrm{~d}, J=11.0 \mathrm{~Hz}, 1 \mathrm{H}, \mathrm{CH}_{2}\right), 2.11\left(\mathrm{~s}, 3 \mathrm{H}, \mathrm{CH}_{3}\right), 1.87\left(\mathrm{~s}, 3 \mathrm{H}, \mathrm{CH}_{3}\right)$, $1.78\left(\mathrm{~d}, J=11.0 \mathrm{~Hz}, 1 \mathrm{H}, \mathrm{CH}_{2}\right), 1.62-1.51(\mathrm{~m}, 1 \mathrm{H}, \mathrm{CH}), 1.10\left(\mathrm{~s}, 3 \mathrm{H}, \mathrm{CH}_{3}\right), 1.08\left(\mathrm{~d}, J=6.7 \mathrm{~Hz}, 3 \mathrm{H}, \mathrm{CH}_{3}\right) .{ }^{13} \mathrm{C}$ NMR (100 MHz, $\left.\mathrm{CDCl}_{3}\right) \delta$ 206.89, 191.67, 165.95, 165.27, 159.13, 143.36, 133.47, 131.31, 127.18, 126.70, 120.13, 112.09, 74.07, 55.90, 46.27, 42.29, 41.17, 31.61, 30.16, 22.59, 22.12, 17.19, 10.74. HR-MS (ESI): Calcd. $\mathrm{C}_{23} \mathrm{H}_{28} \mathrm{O}_{4},[\mathrm{M}+\mathrm{H}]^{+}$m/z: 368.2071, found: 368.2072.

\subsubsection{General Procedure for the Synthesis of Compounds 2a-e}

A mixture of petasin derivatives (1 equiv) which were synthesized as the route II and $\mathrm{m}-\mathrm{ClC}_{6} \mathrm{H}_{5} \mathrm{COOOH}(1.5$ equiv) in $\mathrm{CH}_{2} \mathrm{Cl}_{2}$ was stirred at room temperature for $3 \mathrm{~h}$. The disappearance of petasin derivatives was monitored by TLC. Upon completion, the solvent was removed and water was added, the reaction mixture was extracted with $\mathrm{CH}_{2} \mathrm{Cl}_{2}$. The combined organic layer was washed with brine, dried over anhydrous $\mathrm{MgSO}_{4}$ and concentrated under vacuum to afford the crude product. The crude product was purified by a flash column to yield the pure product 2a-e.

3',3',8,8a-tetramethyl-3-oxo-3,5,6,7,8,8a-hexahydro-1H-spiro[naphthalene-2,2'-oxiran]-7-ylbenzoate (2a). White solid, $90 \%$, mp: $136.1^{\circ} \mathrm{C}-137.6^{\circ} \mathrm{C},{ }^{1} \mathrm{H}$ NMR $\left(400 \mathrm{MHz}, \mathrm{CDCl}_{3}\right) \delta 8.07-7.48$ (m, 5H, ArH), 5.97 (s, $1 \mathrm{H}$, $\mathrm{HC}=\mathrm{C}), 5.14(\mathrm{~m}, 1 \mathrm{H}, \mathrm{CH}), 2.66\left(\mathrm{~d}, J=14.5 \mathrm{~Hz}, 1 \mathrm{H}, \mathrm{CH}_{2}\right), 2.54-2.45\left(\mathrm{~d}, J=14.5 \mathrm{~Hz}, 1 \mathrm{H}, \mathrm{CH}_{2}\right), 2.38(\mathrm{~d}, J=$ 
$\left.11.2 \mathrm{~Hz}, 1 \mathrm{H}, \mathrm{CH}_{2}\right), 2.28-2.15\left(\mathrm{~m}, 2 \mathrm{H}, \mathrm{CH}_{2}\right), 1.89$ (d, $\left.J=11.2,1 \mathrm{H}, \mathrm{CH}_{2}\right), 1.72-1.64(\mathrm{~m}, 1 \mathrm{H}, \mathrm{CH}), 1.48(\mathrm{~s}, 3 \mathrm{H}$, $\left.\mathrm{CH}_{3}\right), 1.33\left(\mathrm{~s}, 3 \mathrm{H}, \mathrm{CH}_{3}\right), 1.30\left(\mathrm{~s}, 3 \mathrm{H}, \mathrm{CH}_{3}\right), 1.05\left(\mathrm{~d}, J=6.7 \mathrm{~Hz}, 3 \mathrm{H}, \mathrm{CH}_{3}\right) .{ }^{13} \mathrm{C} \mathrm{NMR}\left(100 \mathrm{MHz}, \mathrm{CDCl}_{3}\right) \delta$ 194.74, 167.29, 166.14, 133.17, 130.13, 129.59, 129.59, 128.46, 128.46, 125.42, 73.47, 65.79, 65.15, 47.00, 42.12, 40.48, 31.47, 30.48, 21.20, 19.31, 18.93, 10.68. HR-MS (ESI): Calcd. $\mathrm{C}_{22} \mathrm{H}_{26} \mathrm{O}_{4},[\mathrm{M}+\mathrm{H}]^{+} \mathrm{m} / \mathrm{z}$ : 354.1925, found: 354.1924 .

3',3',8,8a-tetramethyl-3-oxo-3,5,6,7,8,8a-hexahydro-1H-spiro[naphthalene-2,2'-oxiran]-7-yl-4-methoxyb enzoate (2b). White solid, yield 92\%, mp: $206.4^{\circ} \mathrm{C}-208.8^{\circ} \mathrm{C},{ }^{1} \mathrm{H}$ NMR $\left(400 \mathrm{MHz}, \mathrm{CDCl}_{3}\right) \delta 8.02-6.95$ (m, $4 \mathrm{H}$, ArH), 5.96 (s, $1 \mathrm{H}, \mathrm{HC}=\mathrm{C}), 5.10(\mathrm{~m}, 1 \mathrm{H}, \mathrm{CH}), 3.89$ (s, 3H, $\left.\mathrm{CH}_{3}\right), 2.66\left(\mathrm{~d}, J=14.5 \mathrm{~Hz}, 1 \mathrm{H}, \mathrm{CH}_{2}\right), 2.49(\mathrm{~d}, J=$ $\left.14.5 \mathrm{~Hz}, 1 \mathrm{H}, \mathrm{CH}_{2}\right), 2.40-2.33\left(\mathrm{~d}, J=11.2 \mathrm{~Hz}, 1 \mathrm{H}, \mathrm{CH}_{2}\right), 2.27-2.08\left(\mathrm{~m}, 2 \mathrm{H}, \mathrm{CH}_{2}\right), 1.87$ (d, $J=11.2 \mathrm{~Hz}, 1 \mathrm{H}$, $\mathrm{CH}_{2}$ ), 1.76 - 1.68 (m, 1H, CH), 1.48 (s, 3H, $\mathrm{CH}_{3}$ ), 1.33 (s, 3H, $\left.\mathrm{CH}_{3}\right), 1.29$ (s, 3H, $\left.\mathrm{CH}_{3}\right), 1.03$ (d, $J=6.7 \mathrm{~Hz}, 3 \mathrm{H}$, $\left.\mathrm{CH}_{3}\right) .{ }^{13} \mathrm{C}$ NMR $\left(100 \mathrm{MHz}, \mathrm{CDCl}_{3}\right) \delta 194.76,167.47,165.89,163.55,131.63,131.63,125.36,122.50,113.70$, 113.70, 73.04, 65.81, 65.14, 55.48, 47.07, 42.13, 40.49, 31.55, 30.51, 21.19, 19.31, 18.93, 10.66. HR-MS (ESI): Calcd. $\mathrm{C}_{23} \mathrm{H}_{28} \mathrm{O}_{5}$, [M+H] $]^{+} \mathrm{m} / \mathrm{z}$ : 384.1995, found: 384.1996.

3',3',8,8a-tetramethyl-3-oxo-3,5,6,7,8,8a-hexahydro-1H-spiro[naphthalene-2,2'-oxiran]-7-yl-2-nitrobenz oate (2c). White solid, yield $91 \%$, mp: $169.8^{\circ} \mathrm{C}-171.7^{\circ} \mathrm{C},{ }^{1} \mathrm{H}$ NMR $\left(400 \mathrm{MHz}, \mathrm{CDCl}_{3}\right) \delta 7.97-7.68(\mathrm{~m}, 4 \mathrm{H}$, ArH), 5.96 (s, 1H, HC=C), 5.15 (m, 1H, CH), 2.63 (d, J = 14.8 Hz, 1H, CH $), 2.52$ (d, J = 14.7 Hz, 1H, CH $)$, 2.47 (d, $\left.J=11.2 \mathrm{~Hz}, 1 \mathrm{H}, \mathrm{CH}_{2}\right), 2.13\left(\mathrm{~m}, 2 \mathrm{H}, \mathrm{CH}_{2}\right), 1.78$ (d, $\left.J=11.2 \mathrm{~Hz}, 1 \mathrm{H}, \mathrm{CH}_{2}\right), 1.70-1.63(\mathrm{~m}, 1 \mathrm{H}, \mathrm{CH})$, $1.48\left(\mathrm{~s}, 3 \mathrm{H}, \mathrm{CH}_{3}\right), 1.32\left(\mathrm{~s}, 3 \mathrm{H}, \mathrm{CH}_{3}\right), 1.29\left(\mathrm{~s}, 3 \mathrm{H}, \mathrm{CH}_{3}\right), 1.04\left(\mathrm{~d}, J=6.7 \mathrm{~Hz}, 3 \mathrm{H}, \mathrm{CH}_{3}\right) .{ }^{13} \mathrm{C} \mathrm{NMR}(100 \mathrm{MHz}$, $\left.\mathrm{CDCl}_{3}\right) \delta 194.62,166.79,165.14,147.90,133.03,131.82,129.58,127.77,125.49,124.01,75.51,65.72,65.13$, 46.56, 42.13, 40.36, 30.65, 30.30, 21.19, 19.27, 18.92, 10.59. HR-MS (ESI): Calcd. $\mathrm{C}_{22} \mathrm{H}_{25} \mathrm{NO}_{6}[\mathrm{M}+\mathrm{H}]^{+} \mathrm{m} / \mathrm{z}$ : 399.2785, found: 399.2783.

3',3',8,8a-tetramethyl-3-oxo-3,5,6,7,8,8a-hexahydro-1H-spiro[naphthalene-2,2'-oxiran]-7-yl-2-fluoroben zoate (2d). White solid, yield $87 \%$, mp: $136.4^{\circ} \mathrm{C}-137.8^{\circ} \mathrm{C},{ }^{1} \mathrm{H}$ NMR $\left(400 \mathrm{MHz}, \mathrm{CDCl}_{3}\right) \delta 7.86-7.32(\mathrm{~m}, 4 \mathrm{H}$, ArH), 5.97 (s, $1 \mathrm{H} \mathrm{HC}=\mathrm{C}), 5.16-5.05(\mathrm{~m}, 1 \mathrm{H}, \mathrm{CH}), 2.66\left(\mathrm{~d}, J=14.4 \mathrm{~Hz}, 1 \mathrm{H}, \mathrm{CH}_{2}\right), 2.46(\mathrm{~d}, J=14.4 \mathrm{~Hz}, 1 \mathrm{H}$, $\mathrm{CH}_{2}$ ), $2.41-2.33$ (d, $\left.J=11.1 \mathrm{~Hz}, 1 \mathrm{H}, \mathrm{CH}_{2}\right), 2.26-2.11\left(\mathrm{~m}, 2 \mathrm{H}, \mathrm{CH}_{2}\right), 1.89\left(\mathrm{~d}, J=11.2 \mathrm{~Hz}, 1 \mathrm{H}, \mathrm{CH}_{2}\right), 1.72-$ $1.63(\mathrm{~m}, 1 \mathrm{H}, \mathrm{CH}), 1.48\left(\mathrm{~s}, 3 \mathrm{H}, \mathrm{CH}_{3}\right), 1.33\left(\mathrm{~s}, 3 \mathrm{H}, \mathrm{CH}_{3}\right), 1.30\left(\mathrm{~s}, 3 \mathrm{H}, \mathrm{CH}_{3}\right), 1.04\left(\mathrm{~d}, J=6.7 \mathrm{~Hz}, 3 \mathrm{H}, \mathrm{CH}_{3}\right) .{ }^{13} \mathrm{C}$ NMR $\left(100 \mathrm{MHz} \mathrm{CDCl}_{3}\right) \delta$ 194.68, 167.0, 164.99, 163.73, 161.27, 132.31, 130.17, 125.34, 120.14, 116.61, 74.01, 65.76, 65.16, 46.91, 42.11, 40.46, 31.39, 30.41, 21.19, 19.29, 18.93, 10.69. HR-MS (ESI): Calcd. $\mathrm{C}_{22} \mathrm{H}_{25} \mathrm{FO}_{4},[\mathrm{M}+\mathrm{H}]^{+} \mathrm{m} / \mathrm{z}: 372.1805$, found: 372.1806 .

3',3',8,8a-tetramethyl-3-oxo-3,5,6,7,8,8a-hexahydro-1H-spiro[naphthalene-2,2'-oxiran]-7-yl-2-fluoroben zoate (2e). White solid, yield $87 \%$, mp: $123.8^{\circ} \mathrm{C}-124.1^{\circ} \mathrm{C},{ }^{1} \mathrm{H}$ NMR $\left(400 \mathrm{MHz}, \mathrm{CDCl}_{3}\right) \delta 7.23-6.87$ (m, $4 \mathrm{H}$, ArH), 5.92 (s, $1 \mathrm{H} \mathrm{HC}=\mathrm{C}), 4.86(\mathrm{~m}, 1 \mathrm{H}, \mathrm{CH}), 3.82$ (s, 3H, $\left.\mathrm{CH}_{3}\right), 3.58\left(\mathrm{~s}, 2 \mathrm{H}, \mathrm{CH}_{2}\right), 2.53(\mathrm{~d}, J=14.4 \mathrm{~Hz}, 1 \mathrm{H}$, $\mathrm{CH}_{2}$ ), 2.46 (d, $\left.J=14.4 \mathrm{~Hz}, 1 \mathrm{H}, \mathrm{CH}_{2}\right), 2.23-2.11$ (d, $\left.J=11.2 \mathrm{~Hz}, 1 \mathrm{H}, \mathrm{CH}_{2}\right), 2.04\left(\mathrm{~m}, 2 \mathrm{H}, \mathrm{CH}_{2}\right), 1.68(\mathrm{~d}, J=11.2$ $\mathrm{Hz}, 1 \mathrm{H}, \mathrm{CH}_{2}$ ), 1.54 - 1.47 (m, 1H, CH), 1.45 (s, 3H, $\left.\mathrm{CH}_{3}\right), 1.30$ (s, 3H, $\left.\mathrm{CH}_{3}\right), 1.21\left(\mathrm{~s}, 3 \mathrm{H}, \mathrm{CH}_{3}\right), 0.86(\mathrm{~d}, J=6.7$ $\left.\mathrm{Hz}, 3 \mathrm{H}, \mathrm{CH}_{3}\right) .{ }^{13} \mathrm{C} \mathrm{NMR}\left(100 \mathrm{MHz}, \mathrm{CDCl}_{3}\right) \delta 194.68,171.53,167.21,158.76,130.19,130.19,125.95,125.35$, 114.03, 114.03, 73.20, 65.74, 65.08, 55.26, 46.73, 42.02, 40.82, 40.39, 31.28, 30.37, 21.16, 19.21, $18.91,10.37$. HR-MS (ESI): Calcd. $\mathrm{C}_{24} \mathrm{H}_{30} \mathrm{O}_{5},[\mathrm{M}+\mathrm{H}]^{+} \mathrm{m} / \mathrm{z}$ : 398.2160, found: 398.2161.

\subsubsection{General Procedure for the Synthesis of Compounds 4a-b}

A mixture of petsasinol (1 equiv) and Maleic anhydride (0.9 equiv) in $\mathrm{CH}_{2} \mathrm{Cl}_{2}$ in the presence of $\left(\mathrm{C}_{2} \mathrm{H}_{5}\right)_{3} \mathrm{~N}(1$ equiv) was stirred at $0^{\circ} \mathrm{C}$ for $2 \mathrm{~h}$. The disappearance of compound petsasinol was monitored by TLC. Upon completion, the solvent was removed and water containing $\mathrm{HCl}$ was added, the reaction mixture was extracted with $\mathrm{CH}_{2} \mathrm{Cl}_{2}$. The combined organic layer was dried over anhydrous $\mathrm{MgSO}_{4}$ and concentrated under vacuum to afford the crude product. The crude product was added to a stirred solution of Excessive halids in the presence of $\mathrm{NaCO}_{3}$ in $\mathrm{DMF}$ at $65^{\circ} \mathrm{C}$. The disappearance of the crude product was monitored by TLC. Upon completion, the solvent was removed and water containing $\mathrm{NaCl}$ was added, the reaction mixture was extracted with EA. The combined organic layer was dried over anhydrous $\mathrm{MgSO}_{4}$ and concentrated under vacuum to afford the crude product. The crude product was purified by a flash column to yield the pure product 4a-b.

allyl (1,8a-dimethyl-6-oxo-7-(propan-2-ylidene)-1,2,3,4,6,7,8,8a-octahydronaphthalen-2-yl)-fumarate (4a). Yellow oil, yield 68\%, ${ }^{1} \mathrm{H}$ NMR $\left(400 \mathrm{MHz}, \mathrm{CDCl}_{3}\right) \delta 6.31$ (d, $\left.J=12.2 \mathrm{~Hz}, 1 \mathrm{H}, \mathrm{HC}=\mathrm{C}\right), 6.28(\mathrm{~d}, J=12.1 \mathrm{~Hz}$, $1 \mathrm{H}, \mathrm{HC}=\mathrm{C}), 5.97(\mathrm{~m}, 1 \mathrm{H}, \mathrm{HC}=\mathrm{C}), 5.80(\mathrm{~s}, 1 \mathrm{H}, \mathrm{HC}=\mathrm{C}), 5.38(\mathrm{~d}, J=17.2 \mathrm{~Hz}, 1 \mathrm{H}, \mathrm{HC}=\mathrm{C}), 5.30(\mathrm{~d}, J=10.4$ $\mathrm{Hz}, 1 \mathrm{H}, \mathrm{HC}=\mathrm{C}), 4.96(\mathrm{~m}, 1 \mathrm{H}, \mathrm{CH}), 4.71\left(\mathrm{~d}, J=5.8 \mathrm{~Hz}, 2 \mathrm{H}, \mathrm{CH}_{2}\right), 2.94\left(\mathrm{~d}, J=13.7 \mathrm{~Hz}, 1 \mathrm{H}, \mathrm{CH}_{2}\right), 2.49(\mathrm{~d}, J=$ 13.6, 4.4 Hz, 1H, $\mathrm{CH}_{2}$ ), $2.34\left(\mathrm{~m}, 2 \mathrm{H}, \mathrm{CH}_{2}\right), 2.21$ (d, $\left.J=11.2 \mathrm{~Hz}, 1 \mathrm{H}, \mathrm{CH}_{2}\right), 2.11\left(\mathrm{~s}, 3 \mathrm{H}, \mathrm{CH}_{3}\right), 1.87\left(\mathrm{~s}, 3 \mathrm{H}, \mathrm{CH}_{3}\right)$, 
$1.74-1.67$ (d, $\left.J=11.2 \mathrm{~Hz}, 1 \mathrm{H}, \mathrm{CH}_{2}\right), 1.51(\mathrm{~m}, 1 \mathrm{H}, \mathrm{CH}), 1.05$ (s, 3H, $\left.\mathrm{CH}_{3}\right), 1.02\left(\mathrm{~d}, J=6.7 \mathrm{~Hz}, 3 \mathrm{H}, \mathrm{CH}_{3}\right) .{ }^{13} \mathrm{C}$ NMR (100 MHz, $\left.\mathrm{CDCl}_{3}\right) \delta 191.64,164.89,164.84,163.42,143.65,131.53,130.02,129.58,127.01,126.76$, 118.97, 75.03, 65.92, 45.99, 42.23, 41.10, 31.23, 29.99, 22.63, 22.14, 17.17, 10.70. HR-MS (ESI): Calcd. $\mathrm{C}_{22} \mathrm{H}_{28} \mathrm{O}_{5},[\mathrm{M}+\mathrm{H}]^{+} \mathrm{m} / \mathrm{z}: 372.2015$, found: 372.2013.

1,8a-dimethyl-6-oxo-7-(propan-2-ylidene)-1,2,3,4,6,7,8,8a-octahydronaphthalen-2-yl-propylfumarate (4b). Yellow oil, yield 69\%, ${ }^{1} \mathrm{H}$ NMR (400 MHz, $\left.\mathrm{CDCl}_{3}\right) \delta 6.29$ (d, $\left.J=12.3 \mathrm{~Hz}, 1 \mathrm{H}, \mathrm{HC}=\mathrm{C}\right), 6.25$ (d, J=12.3 Hz, $1 \mathrm{H}, \mathrm{HC}=\mathrm{C}), 5.80(\mathrm{~s}, 1 \mathrm{H}, \mathrm{HC}=\mathrm{C}), 4.95(\mathrm{~m}, 1 \mathrm{H}, \mathrm{CH}), 4.16\left(\mathrm{t}, J=6.8 \mathrm{~Hz}, 2 \mathrm{H}, \mathrm{CH}_{2}\right), 2.94(\mathrm{~d}, J=13.7 \mathrm{~Hz}, 1 \mathrm{H}$, $\mathrm{CH}_{2}$ ), 2.50 (d, $J=13.7 \mathrm{~Hz}, 1 \mathrm{H}, \mathrm{CH}_{2}$ ), $2.41-2.27$ (m, 2H, $\mathrm{CH}_{2}$ ), 2.21 (d, J = $\left.11.1 \mathrm{~Hz}, 1 \mathrm{H}, \mathrm{CH}_{2}\right), 2.11(\mathrm{~s}, 3 \mathrm{H}$, $\mathrm{CH}_{3}$ ), 1.87 (s, 3H, $\mathrm{CH}_{3}$ ), 1.74 (dd, $J=14.3,7.1 \mathrm{~Hz}, 2 \mathrm{H}, \mathrm{CH}_{2}$ ), $1.69-1.64$ (d, $J=11.1 \mathrm{~Hz}, 1 \mathrm{H}, \mathrm{CH}_{2}$ ), $1.56-1.48$ (m, $1 \mathrm{H}, \mathrm{CH}), 1.05$ (s, 3H, $\left.\mathrm{CH}_{3}\right), 1.02\left(\mathrm{~d}, J=6.7 \mathrm{~Hz}, 3 \mathrm{H}, \mathrm{CH}_{3}\right), 0.98\left(\mathrm{t}, J=7.4 \mathrm{~Hz}, 3 \mathrm{H}, \mathrm{CH}_{3}\right) .{ }^{13} \mathrm{C} \mathrm{NMR}(100$ $\left.\mathrm{MHz} \mathrm{CDCl}_{3}\right) \delta 191.66,171.60,165.32,164.88,143.55,130.10,129.54,127.04,126.76,74.91,66.92,51.64$, 45.99, 42.22, 41.09, 31.23, 30.00, 22.62, 21.81, 20.72, 17.15, 10.70. HR-MS (ESI): Calcd. $\mathrm{C}_{22} \mathrm{H}_{30} \mathrm{O}_{5},[\mathrm{M}+\mathrm{H}]^{+}$ $\mathrm{m} / \mathrm{z}: 374.2174$, found: 374.2172 .

\subsubsection{General Procedure for the Synthesis of Compounds 8a-c}

A mixture of petasinol (1 equiv) and TsOCl (1.5 equiv) in $\mathrm{CH}_{2} \mathrm{Cl}_{2}$ in the presence of $\left(\mathrm{C}_{2} \mathrm{H}_{5}\right)_{3} \mathrm{~N}$ (1 equiv) was stirred at $0^{\circ} \mathrm{C}$ for $6 \mathrm{~h}$. Then the mixture was filtered and concentrated in vacuum. The residue was purified by flash column to yield the pure product 5 . The $\mathrm{NaN}_{3}$ was added to a stirred solution of product 5 in $\mathrm{DMF}$ at $95^{\circ} \mathrm{C}$ for $8 \mathrm{~h}$. The disappearance of product 5 was monitored by TLC. After purification, the product 6 was afforded and was reduced with $\mathrm{Zn}$ power (1 equiv) in the presence of $\mathrm{NH}_{4} \mathrm{Cl}$ (2 equiv) to produce 7. Condensation of product 7 with different kinds of acyl chloride (2 equiv) in $\mathrm{CH}_{2} \mathrm{Cl}_{2}$ in the presence of $\left(\mathrm{C}_{2} \mathrm{H}_{5}\right)_{3} \mathrm{~N}$ (1 equiv) resulted in acyl derivatives. Upon completion, the solvent was removed and water was added, the reaction mixture was extracted with $\mathrm{CH}_{2} \mathrm{Cl}_{2}$. The combined organic layer was dried over anhydrous $\mathrm{MgSO}_{4}$ and concentrated under vacuum to afford the crude product. The crude product was purified by a flash column to yield the pure product 8a-c.

N-(1,8a-dimethyl-6-oxo-7-(propan-2-ylidene)-1,2,3,4,6,7,8,8a-octahydronaphthalen-2-yl)acetamide (8a). Yellow oil, yield 66\%, ${ }^{1} \mathrm{H}$ NMR (400 MHz, $\left.\mathrm{CDCl}_{3}\right) \delta 5.96(\mathrm{~s}, 1 \mathrm{H}, \mathrm{NH}), 5.75$ (s, 1H, HC = C), $4.21(\mathrm{~m}, 1 \mathrm{H}, \mathrm{CH})$, 2.87 (d, $J=14.4 \mathrm{~Hz}, 1 \mathrm{H}, \mathrm{CH}_{2}$ ), 2.44 (d, $J=14.6, \mathrm{~Hz}, 1 \mathrm{H}, \mathrm{CH}_{2}$ ), $2.24-2.18$ (d, $J=11.1 \mathrm{~Hz}, 1 \mathrm{H}, \mathrm{CH}_{2}$ ), $2.15-$ $2.04\left(\mathrm{~m}, 2 \mathrm{H}, \mathrm{CH}_{2}\right), 2.09$ (s, 3H, $\left.\mathrm{CH}_{3}\right), 2.02$ (s, 3H, CH $\mathrm{CH}_{3}, 1.82\left(\mathrm{~s}, 3 \mathrm{H}, \mathrm{CH}_{3}\right), 1.75-1.67(\mathrm{~d}, \mathrm{~J}=11.1 \mathrm{~Hz}, 1 \mathrm{H}$, $\left.\mathrm{CH}_{2}\right), 1.24(\mathrm{~m}, 1 \mathrm{H}, \mathrm{CH}), 1.03\left(\mathrm{~s}, 3 \mathrm{H}, \mathrm{CH}_{3}\right), 1.01\left(\mathrm{~d}, J=6.7 \mathrm{~Hz}, 3 \mathrm{H}, \mathrm{CH}_{3}\right) .{ }^{13} \mathrm{C} \mathrm{NMR}\left(100 \mathrm{MHz}^{\mathrm{CDCl}} \mathrm{CD}_{3} \delta\right.$ 191.44, 169.98, 165.75, 144.53, 127.17, 127.11, 49.39, 43.82, 41.67, 41.03, 30.95, 27.18, 23.77, 22.80, 22.31, 18.18, 12.02. HR-MS (ESI): Calcd. $\mathrm{C}_{17} \mathrm{H}_{25} \mathrm{NO}_{2},[\mathrm{M}+\mathrm{H}]^{+} \mathrm{m} / \mathrm{z}$ : 275.1972, found: 275.1975.

2-chloro-N-(1,8a-dimethyl-6-oxo-7-(propan-2-ylidene)-1,2,3,4,6,7,8,8a-octahydronaphthalen-2-yl)acet amide (8b). Yellow oil, yield 66\%, ${ }^{1} \mathrm{H}$ NMR (400 MHz, $\left.\mathrm{CDCl}_{3}\right) \delta 6.98(\mathrm{~s}, 1 \mathrm{H}, \mathrm{NH}), 5.83(\mathrm{~s}, 1 \mathrm{H}, \mathrm{HC}=\mathrm{C}), 4.20$ (m, 1H, CH), 4.13 (s, 2H, CH $\mathrm{CH}_{2}, 2.93$ (d, $J=14.2 \mathrm{~Hz}, 1 \mathrm{H}, \mathrm{CH}_{2}$ ), 2.47 (d, $J=14.2 \mathrm{~Hz}, 1 \mathrm{H}, \mathrm{CH}_{2}$ ), $2.32-2.23$ (d, $\left.J=11.1 \mathrm{~Hz}, 1 \mathrm{H}, \mathrm{CH}_{2}\right), 2.18-2.03\left(\mathrm{~m}, 2 \mathrm{H}, \mathrm{CH}_{2}\right), 2.13\left(\mathrm{~s}, 3 \mathrm{H}, \mathrm{CH}_{3}\right), 1.85\left(\mathrm{~s}, 3 \mathrm{H}, \mathrm{CH}_{3}\right), 1.75(\mathrm{~d}, J=11.1 \mathrm{~Hz}, 1 \mathrm{H}$, $\left.\mathrm{CH}_{2}\right), 1.26(\mathrm{~m}, 1 \mathrm{H}, \mathrm{CH}), 1.11\left(\mathrm{~s}, 3 \mathrm{H}, \mathrm{CH}_{3}\right), 1.07\left(\mathrm{~d}, J=6.7 \mathrm{~Hz}, 3 \mathrm{H}, \mathrm{CH}_{3}\right) .{ }^{13} \mathrm{C} \mathrm{NMR}\left(100 \mathrm{MHz}^{\mathrm{CDCl}} \mathrm{CD}_{3} \delta\right.$ 191.40, 165.75, 165.17, 144.61, 127.17, 127.06, 50.17, 43.76, 43.01, 41.58, 40.89, 30.75, 27.03, 22.78, 22.26, 17.88, 12.09. HR-MS (ESI): Calcd. $\mathrm{C}_{17} \mathrm{H}_{24} \mathrm{ClNO}_{2}$, [M+H] $]^{+} \mathrm{m} / \mathrm{z}$ : 309.1579, found: 309.1580.

4-chloro-N-(1,8a-dimethyl-6-oxo-7-(propan-2-yli-dene)-1,2,3,4,6,7,8,8a-octahydronaphthalen-2-yl)benza mide (8c). Yellow oil, yield 66\%, ${ }^{1} \mathrm{H}$ NMR (400 MHz, $\left.\mathrm{CDCl}_{3}\right) \delta 7.71-7.45$ (m, 4H, ArH), 6.33 (s, 1H, NH), 5.86 (s, 1H, HC = C), 4.43 (m, 1H, CH), 2.95 (d, J = $\left.14.2 \mathrm{~Hz}, 1 \mathrm{H}, \mathrm{CH}_{2}\right), 2.49$ (d, J = 14.2 Hz, 1H, CH $), 2.33$ 2.28 (d, $J=11.1 \mathrm{~Hz}$ ), $2.22-2.19\left(\mathrm{~m}, 2 \mathrm{H}, \mathrm{CH}_{2}\right), 2.15$ (s, 3H, $\mathrm{CH}_{3}$ ), 1.92 (d, $J=11.3 \mathrm{~Hz}$ ), 1.87 (s, 3H, $\mathrm{CH}_{3}$ ), $1.31-1.25(\mathrm{~m}, 1 \mathrm{H}, \mathrm{CH}), 1.17\left(\mathrm{~s}, 3 \mathrm{H}, \mathrm{CH}_{3}\right), 1.13\left(\mathrm{~d}, J=6.7 \mathrm{~Hz}, 3 \mathrm{H}, \mathrm{CH}_{3}\right) .{ }^{13} \mathrm{C} \mathrm{NMR}\left(100 \mathrm{MHz}^{\mathrm{CDCl}} \mathrm{CD}_{3} \delta\right.$ 191.37, 166.38, 165.29, 144.75, 137.92, 133.09, 131.46, 129.05, 128.77, 128.13, 127.24, 127.06, 50.12, 44.03, 41.59, 41.05, 30.89, 27.20, 22.83, 22.32, 18.29, 12.18. HR-MS (ESI): Calcd. $\mathrm{C}_{22} \mathrm{H}_{26} \mathrm{ClNO}_{2},[\mathrm{M}+\mathrm{H}]^{+} \mathrm{m} / \mathrm{z}$ : 371.1710, found: 371.1708.

\subsection{Bioassays}

\subsubsection{Grow Inhibition Assay}

The petasin derivatives were assayed for in vitro anticancer activity by using MTT method. Cells were seeded in 96-well plates at a density of $4.6 \times 10^{3}$ cells/well for $24 \mathrm{~h}$. The cancer cell lines included SK-N-SH, MGC-803 
and HepG-2. The seeded cells were treated with various concentrations of the test derivatives for $48 \mathrm{~h}$. After 20 $\mathrm{ml}$ of MTT (3-(4, 5-dimethylthiazol-2-yl)-2, 5-diphenyltetrazolium bromide) solution (5 mg/mL) was added to each well and incubated for $4 \mathrm{~h}$ at $37^{\circ} \mathrm{C}$. The medium containing MTT was discarded; then $150 \mathrm{~mL}$ of dimethyl sulfoxide (DMSO) was added to each well. And the plates were agitated until the dark blue crystals (formazan) had completely dissolved. The absorbance was measured using a microplate reader at a wavelength of $490 \mathrm{~nm}$. The average $50 \%$ inhibitory concentration $\left(\mathrm{IC}_{50}\right)$ was determined from the concentration response curves according to the inhibition ratio for each concentration.

\subsubsection{Statistical Analysis}

Cell activity data were analysed by the SPSS 17.0 software and were expressed as means \pm standard error (SE).

\section{Acknowledgements}

The authors are grateful for the financial support from the National Natural Science Foundation of China (No. 81273393).

\section{References}

[1] Berbegall, A.P., Villamon, E., Tadeo, I., Martinsson, T., Canete, A., Castel, V., Navarro, S. and Noguera, R. (2014) Neuroblastoma after Childhood: Prognostic Relevance of Segmental Chromosome Aberrations, ATRX Protein Status, and Immune Cell Infiltration. Neoplasia, 16, 471-480. http://dx.doi.org/10.1016/j.neo.2014.05.012

[2] Agarwala, S., Mandelia, A., Bakhshi, S., Srinivas, M., Bajpai, M., Gupta, A.K., Gupta, D.K. and Bhatnagar, V. (2014) Neuroblastoma: Outcome over a 14 Year Period from a Tertiary Care Referral Centre in India. Journal of Pediatric Surgery, 49, 1280-1285. http://dx.doi.org/10.1016/j.jpedsurg.2014.03.017

[3] Spix, C., Pastore, G., Sankila, R., Stiller, C.A. and Steliarova-Foucher, E. (2006) Neuroblastoma Incidence and Survival in European Children (1978-1997): Report from the Automated Childhood Cancer Information System Project. European Journal of Cancer, 42, 2081-2091. http://dx.doi.org/10.1016/j.ejca.2006.05.008

[4] Cheng, Y.F., Wang, C.F., Li, W.J., Lin, Q. and Zhang, Q. (2012) Antiproliferation Effects of Petasin on Human Neuroblastoma SK-N-SH Cells. Acta Neuropharmacologica, 1, 1-6.

[5] Yi, W.-B., Zhu, Y.-W. and Cai, C. (2013) Fluorous 4-N,N-Dimethylaminopyridium Iodide: Recyclable Organocatalysts by Precipitation for Acylation Reaction at Room Temperature. Catalysis Communications, 41, 26-28. http://dx.doi.org/10.1016/j.catcom.2013.06.031

[6] Liu, Z., Ma, Q., Liu, Y. and Wang, Q. (2014) 4-(N,N-Dimethylamino)Pyridine Hydrochloride as a Recyclable Catalyst for Acylation of Inert Alcohols: Substrate Scope and Reaction Mechanism. Organic Letters, 16, 236-239. http://dx.doi.org/10.1021/ol4030875

[7] Ozaki, A., Sumita, K., Goto, K. and Matsumoto, A. (2013) Synthesis of Poly(Decahydro-2-Naphthyl Methacrylate)s with Different Geometric Structures and Effects of Side-Group Dynamics on Polymer Properties Investigated by Thermal and Dynamic Mechanical Analyses and DFT Calculations. Macromolecules, 46, 2941-2950. http://dx.doi.org/10.1021/ma400254d

[8] Vyas, D.J., Larionov, E., Besnard, C., Guenee, L. and Mazet, C. (2013) Isomerization of Terminal Epoxides by a [Pd-H] Catalyst: A Combined Experimental and Theoretical Mechanistic Study. Journal of the American Chemical Society, 135, 6177-6183. http://dx.doi.org/10.1021/ja400325w

[9] Hyun, M.Y., Kim, S.H., Song, Y.J., Lee, H.G., Jo, Y.D., Kim, J.H., Hwang, I.H., Noh, J.Y., Kang, J. and Kim, C. (2012) Terminal and Internal Olefin Epoxidation with Cobalt(II) as the Catalyst: Evidence for an Active Oxidant Co(II)-Acylperoxo Species. The Journal of Organic Chemistry, 77, 7307-7312. http://dx.doi.org/10.1021/jo3009963

[10] Elbert, B.L., Lim, D.S., Gudmundsson, H.G., O’Hanlon, J.A. and Anderson, E.A. (2014) Synthesis of Cyclic Alkenylsiloxanes by Semihydrogenation: A Stereospecific Route to (Z)-Alkenyl Polyenes. Chemistry, 20, 8594-8598. http://dx.doi.org/10.1002/chem.201403255

[11] Maury, J., Feray, L., Bertrand, M.P., Kapat, A. and Renaud, P. (2012) Unexpected Conversion of Alkyl Azides to Alkyl Iodides and of Aryl Azides to N-Tert-Butyl Anilines. Tetrahedron, 68, 9606-9611. http://dx.doi.org/10.1016/j.tet.2012.09.066

[12] Juríček, M., Felici, M., Contreras-Carballada, P., Lauko, J., Bou, S.R., Kouwer, P.H.J., Brouwer, A.M. and Rowan, A.E. (2011) Triazole-Pyridine Ligands: A Novel Approach to Chromophoric Iridium Arrays. Journal of Materials Chemistry, 21, 2104-2111. http://dx.doi.org/10.1039/C0JM03117H

[13] Ogawa, S., Asada, M., Ooki, Y., Mori, M., Itoh, M. and Korenaga, T. (2005) Design and Synthesis of Glycosidase Inhibitor 5-Amino-1,2,3,4-cyclohexanetetrol Derivatives from (-)-Vibo-Quercitol. Bioorganic \& Medicinal Chemistry, 
13, 4306-4314. http://dx.doi.org/10.1016/j.bmc.2005.04.003

[14] Reed, C.S., Huigens III, R.W., Rogers, S.A. and Melander, C. (2010) Modulating the Development of E. coli Biofilms with 2-Aminoimidazoles. Bioorganic \& Medicinal Chemistry Letters, 20, 6310-6312. http://dx.doi.org/10.1016/j.bmcl.2010.08.075

[15] Ma, L., Li, S., Zheng, H., Chen, J., Lin, L., Ye, X., Chen, Z., Xu, Q., Chen, T., Yang, J., Qiu, N., Wang, G., Peng, A., Ding, Y., Wei, Y. and Chen, L. (2011) Synthesis and Biological Activity of Novel Barbituric and Thiobarbituric Acid Derivatives against Non-Alcoholic Fatty Liver Disease. European Journal of Medicinal Chemistry, 46, 2003-2010. http://dx.doi.org/10.1016/j.ejmech.2011.02.033

[16] Kumar, B.R.P. and Nanjan, M.J. (2010) Novel Glitazones: Design, Synthesis, Glucose Uptake and Structure-Activity Relationships. Bioorganic \& Medicinal Chemistry Letters, 20, 1953-1956. http://dx.doi.org/10.1016/j.bmcl.2010.01.125

[17] Zhou, L. and Lu, W. (2014) Palladium-Catalyzed Beta-Acyloxylation of Simple Amide via sp ${ }^{3}$ C-H Activation. Organic Letters, 16, 508-511. http://dx.doi.org/10.1021/ol403393w

[18] Jin, Y., Chen, S.W. and Tian, X. (2006) Synthesis and Biological Evaluation of New Spin-Labeled Derivatives of Podophyllotoxin. Bioorganic \& Medicinal Chemistry, 14, 3062-3068. http://dx.doi.org/10.1016/j.bmc.2005.12.025

[19] Perez-Areales, F.J., Di Pietro, O., Espargaro, A., Vallverdu-Queralt, A., Galdeano, C., Ragusa, I.M., Viayna, E., Guillou, C., Clos, M.V., Perez, B., Sabate, R., Lamuela-Raventos, R.M., Luque, F.J. and Munoz-Torrero, D. (2014) Shogaol-Huprine Hybrids: Dual Antioxidant and Anticholinesterase Agents with $\beta$-Amyloid and Tau Anti-Aggregating Properties. Bioorganic \& Medicinal Chemistry, 22, 5298-5307. http://dx.doi.org/10.1016/j.bmc.2014.07.053

[20] Kawano, H., Shakushiro, K., Nakata, M., Kita, A., Maeda, A., Watanabe, S., Sako, K. and Oku, N. (2014) Antitumor Efficacy and Biodistribution of Liposomal Sepantronium Bromide (YM155), a Novel Small-Molecule Survivin Suppressant. European Journal of Pharmaceutics and Biopharmaceutics, 88, 283-289. http://dx.doi.org/10.1016/j.ejpb.2014.06.015

[21] Rodriguez-Berna, G., Mangas-Sanjuan, V., Gonzalez-Alvarez, M., Gonzalez-Alvarez, I., Garcia-Gimenez, J.L., Diaz Cabanas, M.J., Bermejo, M. and Corma, A. (2014) A Promising Camptothecin Derivative: Semisynthesis, Antitumor Activity and Intestinal Permeability. European Journal of Medicinal Chemistry, 83, 366-373. http://dx.doi.org/10.1016/j.ejmech.2014.06.050

[22] Zhang, D., Debnath, B., Yu, S., Sanchez, T.W., Christ, F., Liu, Y., Debyser, Z., Neamati, N. and Zhao, G. (2014) Design and Discovery of 5-Hydroxy-6-Oxo-1,6-Dihydropyrimidine-4-Carboxamide Inhibitors of HIV-1 Integrase. Bioorganic \& Medicinal Chemistry, 22, 5446-5453. http://dx.doi.org/10.1016/j.bmc.2014.07.036 